\title{
Arbor
}

\section{Investigación y servicios de información en Colombia}

\section{Alfonso Monsalve Solórzano}

Arbor CLVII, 617-618 (Mayo-Junio 1997) 133-146 pp.

En primer lugar, presentaré brevemente un panorama del lugar que ocupa la investigación científica en Colombia. Luego, expondré las características del Sistema Nacional Ciencia y Tecnología y las políticas gubernamentales actuales sobre investigación científica, y alli enfatizaré el papel del sistema nacional de información. Después, resaltaré algunas acciones en el campo internacional y finalmente haré una apreciación sobre el potencial que pueden ofertar las bibliotecas especializadas (universitarias y de centros de investigación) para establecer programas coordinados que generen cauces de cooperación, lo cual se traduciría en un instrumento enormemente útil para la investigación.

\section{Introducción}

Los países latinoamericanos, a diferencia de los países miembros de la UE, no estamos integrados en un sistema global de $\mathrm{I}+\mathrm{D}$ que marque unas directrices para todos los países miembros. Por eso, aunque los esfuerzos orientados a la coordinación entre nuestras políticas científicas son cada vez mayores y son múltiples las acciones concertadas, voy a centrarme exclusivamente en el caso colombiano (es decir, un enmarque y una justificación de su posicionamiento para clarificar a los lectores).

\section{El lugar de la investigación científica en Colombia}

El diagnóstico presentado por el Consejo Nacional de Ciencia y Tecnología sobre el estado de la investigación científica en Colombia 


\section{Alfonso Monsalve Solórzano}

y la política en este campo para los años 1994-1998 (1995a, p.8), es indicativo de las dificultades pero también de las expectativas y posibilidades que este país tiene en esta actividad.

Se trata de una nación con una economía intermedia, que posee, no obstante, uno de los más bajos índices de científicos de acuerdo con su nivel de desarrollo. Así, Colombia tiene unos 4500 científicos, de los cuales la mitad no han realizado estudios de maestría o doctorado. Esto equivale a una proporción de 166 científicos por millón de habitantes, mientras que naciones como Brasil, Argentina, Chile y México cuentan con 400 científicos por millón ${ }^{1}$.

En su cojunto, los datos de Latinoamérica son altamente preocupantes si se tiene en cuenta que Estados Unidos y Japón tienen 2900 y 3500 investigadores por millón de habitantes, respectivamente. Los indicadores de la producción científica reflejan, también, la situación descrita: América Latina participa con el 1\% de las publicaciones de este tipo en el mundo y Colombia produce el $1 \%$ de las publicaciones de la región.

No obstante, el país es cada vez más consciente de la necesidad de invertir en investigación científica, y pese a los problemas económicos y a prioridades que exigen atención inmediata, la inversión en términos del Producto Interno Bruto ha crecido, aunque no en la proporción deseada: en los inicios de la década de los 90 se dedicaba sólo el $0.3 \%$ del PIB a esta clase de investigación, en 1995 aumentó al $0.5 \%$. Para 1997 está prevista una inversión del $0.87 \%$ del PIB, es decir 100.8539 millones de pesos (unos $132.702,5$ millones de pesetas) y para 1998 está previsto que sea del 1\%, o sea, 1071539 millones de pesos (140.992 millones de pesetas, aproximadamente), equivalente a la media de los países latinoamericanos. Pero este aumento es aún muy bajo si se tiene en cuenta que en los países industrializados invierten entre el 2 y el $4 \%$ del PIB a esta actividad.

La investigación científica tiene en Colombia 3 fuentes de financiación: los recursos del presupuesto nacional asignados a instituciones y universidades públicas, los provenientes del sector privado y los que se canalizan a través de los Programas Nacionales de Ciencia y Tecnología, procedentes del presupuesto nacional o del crédito externo. Por otra parte, es importante resaltar que en Colombia existe infraestructura y tradición investigativa importante en alguna áreas. Así por ejemplo, en el sector agropecuario (en el que el estado invierte el $45 \%$ de su inversión en investigación), instituciones del estado como el Instituto Colombiano Agropecuario, ICA, han hecho aportes significativos en el mejoramiento de semillas; y centros de investigación privados 
como el Centro de Investigaciones del Café, CENICAFÉ, y el Centro de Investigaciones de la Caña de Azúcar, CENICAÑA, han logrado éxitos importantes en sus respectivos campos (como la creación de una variedad de café resistente a la roya). En el área de las ciencias básicas biomédicas, logros como los de la vacuna contra la malaria, diseñada por el grupo encabezado por el doctor Manuel Elkin Patarroyo, son de reconocimiento mundial.

\section{El Sistema Nacional de Ciencia y Tecnología}

En realidad, la investigación científica y tecnológica forma parte desde hace tiempo de los planes de desarrollo nacional. Su actual marco legal es la Constitución Política de 1991, la Ley 29 de 1990 (que dicta normas para el fomento de la investigación), y los Decretos Ley 393 (que normatiza las formas de asociación para la investigación), 584 (que reglamenta los viajes al exterior de los investigadores), 585 (por el que se reestructura COLCIENCIAS), y 591 (por el cual se normatizan las modalidades de contratos de fomento de la investigación) de 1991, entre otros.

El Sistema Nacional de Ciencia y Tecnología (SNCyT) ha venido consolidándose de manera progresiva. Un paso clave ha sido, precisamente, adscribir al Instituto Colombiano para el Desarrollo de la Ciencia y la Tecnología "Francisco José de Caldas", COLCIENCIAS, el organismo gubernamental encargado de planificar, ejecutar y coordinar las políticas de investigación, al Departamento Nacional de Planeación (anteriormente pertenecía al Ministerio de Educación).

Otro, consistió en la creación o consolidación del Consejo Nacional de Ciencia y Tecnología, (conformado por organizaciones del estado vinculadas a la planeación económica y a las políticas de producción, al sector privado y a las actividades académicas e investigativas) y de los siguientes once (11) programas nacionales estructurados por áreas de saber o por sectores de la producción, gestionados por sus respectivos Consejos De Programa, quienes administran las políticas nacionales de investigación en los sectores de su competencia:

- Programa Nacional de Ciencia y Tecnología de la salud.

- Programa Nacional de Ciencias Básicas.

- Programa Nacional de Investigaciones en Energía y Minería.

- Programa Nacional de Ciencia y Tecnología Agropecuaria.

- Programa Nacional de Desarrollo Tecnológico Industrial y Calidad. 
- Programa Nacional de Ciencia y Tecnología del Mar.

- Programa Nacional de Ciencias Sociales y`Humanas.

- Programa Nacional de Estudios Científicos en Educación.

- Programa Nacional de Biotecnología.

- Programa Nacional del Medio Ambiente y Hábitat.

- Programa Nacional de Electrónica, Informática y Telecomunicaciones.

También se crearon dentro del Sistema Nacional seis (6) Comisiones Regionales de Ciencia y Tecnología, una por cada región histórico-cultural, con el fn de descentralizar -en un país con tendencias centralistas muy fuertes- la investigación.

Finalmente, se ha avanzado en la construcción de una red telemática nacional que hace parte de la red mundial de redes. Más adelante se explicitará este punto.

\section{El Sistema Nacional de Información y las Bibliotecas Universitarias}

El país viene desarrollando esfuerzos en estas áreas desde hace ya bastante tiempo.

En cuanto a la telemática, las Universidades Nacional (pública), de los Andes y del Norte (privadas) iniciaron pruebas para conectarse internacionalmente. En 1990 el Instituto Colombiano para el Fomento de la Educación Superior, ICFES, organismo estatal encargado del control de las universidades colombianes, creó la Red Universitaria Nacional de Colombia, RUNCOL, la cual enlazó internacionalmente, a través de la Universidad de los Andes, con la red BITNET. La conexión nacional se hizo vía conexiones telefónicas convencionales y Coldaq, un servicio ofrecido por la Empresa Nacional de Telecomunicaciones, TELECOM.

En 1994, COLCIENCIAS y el ICFES acuerdan constituir la Red de Ciencia, Educación y Tecnología de Colombia, CETCOL, con el fin de "dotar al país de una infraestructura de comunicación de datos que sea común al sistema Nacional de Ciencia y Tecnología, al Sistema Nacional de Información sobre Educación Superior....y establecer una red colombiana cooperativa de transmisión de datos, dentro de las reglas y protocolos de Internet y su conexión internacional; facilitar la interconexión nacional y motivar el desarrollo de las redes institucionales y regionales" (ICFES, 1994). 
En las redes de bibliotecas y centros de información, se viene trabajando desde mediados de la década del 60 cuando la Asociación Colombiana de Universidades, ASCUN y el Fondo Universitario Nacional, Fun, reglamentaron el Grupo de Trabajo para Bibliotecas Universitarias. Este organismo se convirtió, en 1968, en el Consejo Nacional de Servicios Bibliotecarios, bajo la coordinación del Instituto Colombiano para el Fomento de la Educación Superior, ICFES, organismo gubernamental para la política universitaria colombiana, creado ese año a partir del FUN. El Consejo estudió la situación de esta clase de bibliotecas, determinó las necesidades esenciales, elaboró un plan de desarrollo y elaboró las normas generales de su funcionamiento.

En 1972 el Consejo fué reemplazado por los Comités Regionales de Bibliotecas Universitarias, coordinados por la División de Recursos Bibliográficos del ICFES. En 1977 se establece, bajo el control de ese organismo, la Red Colombiana de Bibliotecas de Educación Superior, y en 1983, el ICFES convierte esa Red en el Sistema de Información y Documentación para la Educación Superior, SIDES, como subsistema del Sistema Nacional de Información, SNI. (Cardona de Gil y Campuzano 1991, p. 130). El SIDES tiene 3 componentes (ibid, p.132 y ss.), constitutivos ellos de una red: Un nodo central, coordinador, en la Hemeroteca Nacional del ICFES, en Santafé de Bogotá, 9 nodos especializados, y las unidades de información de la educación superior, agrupadas regionalmente.

Los nodos especializados están ubicados en bibliotecas de universidades que son fuertes en áreas específicas. Los nodos son:

- Ciencias de la salud: Universidad de Antioquia, en Medellín.

- Ingenierías: Universidad Industrial de Santander, en Bucaramanga.

- Ciencias de la Educación: Universidad Pedagógica Nacional, Santafé de Bogotá.

- Ciencias básicas: Universidad del Valle, Cali.

- Ciencias Sociales: Universidad Nacional de Colombia, sede Santafé de Bogotá.

- Ciencias agrícolas: Universidad Nacional de Colombia, sede Medellín.

- Derecho y Ciencias Políticas: Universidad Externado de Colombia, Santafé de Bogotá.

- Ciencias Económicas: Universidad de los Andes, Santafé de Bogotá.

- Transportes, vías y telecomunicaciones: Universidad del Cauca, Popayan. 
Asímismo, se constituyeron los Comités Regionales de Directores de Bibliotecas, agrupados en 6 zonas geográficas del país.

Lo que se pretendió con el SIDES fue racionalizar los recursos de adquisiciones bibliográficas, en especial, revistas científicas, concentrándolos en esas universidades para que circularan nacionalmente gracias a la red. Pero por distintas causas, este proyecto no ha funcionado a plenitud. No obstante, algunos de los Comités Regionales han continuado operando y prestando servicios importantes en su área de influencia. Además, ofreció algunos productos y servicios que sí lo hicieron y hoy son una parte constitutiva básica de los recursos de información para la investigación científica del país. Entre ellos cabe señalar:

- El Catálogo Colectivo Nacional de Publicaciones Seriadas. Allí se encuentran registradas todas las publicaciones de ese tipo, nacionales y extranjeras a partir de 1971 , que poseen las bibliotecas universitarias colombianas, la Biblioteca Nacional y la Biblioteca Luis Angel Arango del Banco de la República (banco central del país), que acaba de poner su catálogo en el ciberespacio). El Catálogo Colectivo está en Internet y presta servicio de fotocopia de artículos a cualquier parte del territorio Nacional.

- Banco Nacional de Analíticas, que contiene información sobre revistas científicas latinoamaricanas, con énfasis en Colombia. En la actualidad está desactualizado.

- Banco Nacional de tesis.

\section{Políticas actuales}

El Consejo Nacional de Política Económica y Social (Compes), elaboró la POLÍTICA NACIONAL DE CIENCIA Y TECNOLOGÍA para los años 1994-1998 (1994). Posteriormente, El Consejo Nacional de Ciencia y Tecnología, a través de Colciencias, (1995a, citado más arriba) presentó el ya mencionado documento CIENCIA Y TECNOLOGIA PARA UN DESARROLLO SOSTENIBLE Y EQUITATIVO. Implementación de la Política Nacional de Ciencia y Tecnología: 1994-1998, y COLCIENCIAS puso a consideración (1995b) y ya se está ejecutando el Plan Estratégico de los Sistemas de Información Científica y Tecnológica.

El objetivo general de la política de investigación en el país es, en el espíritu de los artículos 1-3 de la mencionada ley 29 de 1990, «integrar la ciencia y la tecnología a los diversos sectores de la vida nacional, buscando incrementar la competitividad del sector productivo, en el contexto de una política de internacionalización de la economía, 
El papel de la Biblioteca Nacional en la Investigación

y mejorar el bienestar y la calidad de vida de la población colombiana" (1995a, p.11).

Para logar ese objetivo se impulsan cinco estrategias, (ibid, p.12):

E1. «Desarrollar y fortalecer la capacidad nacional en ciencia y tecnología». Esto implica la formación de recursos humanos altamente cualificados y el apoyo financiero a la investigación.

E2. "Crear condiciones de competitividad en el sector productivo nacional por medio de políticas sectoriales activas que contemplen la tecnología como factor crucial para el desarrollo de ventajas competitivas adquiridas». Esto significa creación de redes de innovación que vinculen los centros productivos con los centros de investigación y la creación de un Sistema Nacional de Innovación.

E3. «Fortalecer la capacidad para mejorar los servicios sociales y generar conocimiento sobre la realidad social de país».

E4. "Generar y aplicar conocimiento científico orientado a asegurar un desarrollo sostenible».

E5. "Integrar la ciencia y la tecnología a la sociedad y a la cultura colombianas».

Estas estrategias se desglosan en ocho objetivos específicos, de los cuales citaré los que interesan en este trabajo:

OE1. La creación y consolidación de gupos y centros de investigación

OE3. La formación de recursos humanos.

Para alcanzar éstos (y los otros seis objetivos no mencionados), se están desarrollando programas y líneas de acción de los cuales creo importante destacar:

PL1. La Política de Formación en Recursos Humanos.

- Una meta es apoyar en el lapso de los cuatro años (1994-1998) la formación de 2000 personas a nivel de posgrado, especialmente en doctorado, en el país y el exterior. Existe un programa de ochocientas becas, administrado por Colciencias, el Progama Banco Interamericano de Desarrollo (BID) - Colciencias -3, en el que los postulantes concursan rigurosamente por méritos. Estas becas cubren costos de académicos y de manutención de los beneficiarios. Hay además instituciones privadas y mixtas, como Colfuturo, que desarrollan programas propios.

- Se pretende además mejorar y actualizar la formación de los recursos humanos ya vinculados a la investigación. El programa BID - Colciencias -3, está financiando 300 pasantías y 70 cursos de actualización y entrenamiento, ofreciando este tipo de capacitación a 1.400 personas aproximadamente. En caso de grupos 
de investigación, esta modalidad financia becas post-doctorales a investigadores de trayectoria.

- Consolidar los doctorados existentes mediante el Programa de Apoyo Institucional a Posgrados Nacionales.

- El Programa de Jóvenes Investigadores estimula la formación no conducente a título a personas jóvenes con potencial investigativo.

- Fortalecimiento al programa Estímulo a Investigadores. En 1994 se seleccionaron 153 investigadores, en 1995a, 250 que reciben estímulos monetarios importantes durante un año, renovable indefinidamente, a condición de que permanezcan en la actividad investigativa y publiquen libros o escriban en revistas de investigación.

- Fortalecimiento del Programa de Movilidad de Científicos, cuya finalidad es vincular este tipo de personas con formación doctoral, extranjeros o colombianos residentes por más de cinco años en el extranjero, a los centros de investigación y a las empresas que desarrollan investigación.

PL2. Apoyo a la Investigación y Consolidación de Grupos y Centros de Investigación.

Se pretende crear y consolidar grupos de excelencia en ciencias básicas y sociales. De las 150 instituciones de nivel universitario en 1995, no más de 8 tienen una importante actividad investigativa. Esta es una situación que se debe revertir. Pero, además, los grupos deben alcanzar niveles internacionales de producción científica, así como sus mecanismos de difusión. Para esto en (1995a, pp. 33 y siguientes) se han fijado las siguientes metas en las cuales se ha venido trabajando seriamente

- Apoyo financiero a grupos y centros de excelencia necesarios para el país. En este campo cabe destacar la colaboración de la Unión Europea a través de la Red Alfa. En Colombia existe un Comité Nacional de Red Alfa. Una de las características de este Programa es que además de implicar a investigadores de por lo menos 3 estados miembros de la UE, integra grupos de investigación de distintos países latinoamericanos.

- Apoyo a publicaciones científicas, tratando de tener al menos 12 revistas en los índices internacionales en los distintos campos o disciplinas.

- Apoyo para infraestructura a programas de doctorado. Entre el 94 y el 98 se financiará infraestructura a por lo menos 15 de estos programas.

PL3. Apoyo a la innovación, competitividad y desarrollo tecnológico. 
El papel de la Biblioteca Nacional en la Investigación

- El país, consciente del papel que en el desarrollo de las fuerzas productivas cumplen el conociemiento científico y tecnológico está impulsando el Sistema Nacional de Innovación apara apoyar la modernización y reconversión tecnológica de las empresas e incrementar la inversión de éstas en el campo investigativo, entre otros objetivos. Se espera en el cuatrenio 1994-1998, beneficiar a más de 4.000 empresas, estableciendo o reforzando Centros Tecnológicos Sectoriales y Regionales., de los cuales ya hay 14 establecidos. Durante ese período COLCIENCIAS y el Instituto de Fomento Industrial (IFI), financiarán más de 200 proyectos de innovación tecnológica.

- Se moderniza el sistema de financiamiento para la innovación tecnológica mediante las modalidades de cofinanciación, riesgo compartido, financiamiento directo, capital semilla y garantías para la innovación.

PL3. Fortalecimiento del Sistema Nacional de Información, SNI.

Para conseguir que el Sistema de Información funcione adecuadamente se ha constituido una estructura operativa que permita la agilidad en la toma de decisiones y la cooperación entre las entidades que estén involucradas en él. Para ello, (COLCIENCIAS, 1995a, p. 11) el Sistema tendrá la siguiente organización:

- Un Consejo Nacional conformado por los directores de COLCIENCIAS, ICFES y Departamento Administrativo Nacional de Estadísticas, DANE y representantes del sector académico, investigativo y empresarial.

- Un comité Asesor, integrado por funcionarios de COLCIENCIAS, ICFES y DANE, y representantes del sector académico, investigativo y empresarial. COLCIENCIAS asume la secretaría técnica del SNI. Este es un cambio importante porque, como se vió anteriormente, era el ICFES, quien coordinaba el SNI.

- Una Secretaría Ejecutiva, que pertenecerá a una de las instituciones y que se rotará cada 2 años.

- La Corporación InterRed, de la que se hablará más abajo.

De acuerdo con (ibid, pp. 65 y ss.), Colombia debe enfrentar 2 retos en este campo: "...el primero consiste en el desarrollo de una Politica Nacional de Información sólida, consistente y relacionada con temas como el acceso universal a la información, normatividad, interoperabilidad, privacidad, propiedad intelectual y conservación del conocimiento autónomo...El segundo de los retos consiste en la ampliación y consolidación de la Infraestructura Nacional de Información». Esta infraestructura consta de 3 niveles: 
- La consolidación de bases de datos especializadas.

- La interrelación entre esas bases para construir redes de información.

- La implementación de la infraestructura telemática que se requiere para interconectar entidades, servicios de información y bases.

La meta más importante es el fortalecimieto de la infraestructura nacional de información. Para ello,

- Se desarrollan Sistemas Especializados de información en sectores como las ciencias básicas, medio ambiente, económico-social, energético, etc.

Hasta el momento, COLCIENCIAS ha apoyado, entre otros, los siguientes sistemas y bases de datos (Colciencias 1996):

1. Tema: Economía

Acción: servicio de información.

Nombre: Accesibilidad de la Base de Datos de Economía a través de Internet.

Ejecutor: Universidad de los Andes.

Nombre: Banco de Datos Estadísticos de Consulta Remota.

Ejecutor: CEGA.

2. Tema: Salud.

Acción: sistema de información.

Nombre: Red Colombiana de Informática en Salud y Ciencias Biomédicas.

Ejecutor: Federación Panamericana de Asociaciones de Facultades (escuelas) de Medicina, FEPAMEM..

3. Tema: Infraestructura telemática.

Acción: interconexión.

Nombre: Red Nacional de Centros de Gestión.

Ejecutores: Universidades dell Cauca., Caldas, Francisco de Paul Santander y de la Guajira.

4. Tema: Agropecuaria.

Acción: sistema de información.

Nombre: Red de Centros de Información y Documentación para el Sector Agropecuario.

Ejecutor: Corporación Colombiana de Investigación Agropecuaria, CORPOICA.

5. Tema: Educación.

Acción: aplicación de tecnologías de información.

Nombre: Centro Linux.

Ejecutor: Universidad del Valle. 


\section{El papel de la Biblioteca Nacional en la Investigación}

6. Tema: Industria.

Acción: servicio de información.

Nombre: Red de Información técnica - ICONTEC.

Ejecutor: Instituto Colombiano de Normas Técnicas y Certificación, ICONTEC.

Nombre: Integración del SIE a la Red CETCOL.

Ejecutor: INNOTECH.

7. Tema: Políticas de Información.

Nombre: Políticas de Inversión en Informática en el Sector Público.

Ejecutor: Departamento Nacional de Planeación, DNP - COLCIENCIAS - Departamento Nacional de Estadísticas, DANE.

Nombre: Tendencias de la Tecnología de Información e Impacto en Países en Desarrollo. Ejecutor: COLCIENCIAS.

8. Tema: General

Acción: sistema de información.

Nombre: Sistema de Información de Arqueológía Colombiana.

Ejecutor: Fundación Erigaie.

Nombre: Observatorios Ambientales Urbanos.

Ejecutor: Ministerio del Medio Ambiente.

Nombre: Sistema de información Minera - Energética -SIMEC.

Ejecutor: Ministerio de Minas y Energía.

Nombre: Datos Arqueológicos de la Región del Caribe.

Ejecutor: Universidad del Norte.

Servicio de Información de Estadística del DANE en Línea.

Ejecutor: DANE.

Nombre: Sistema de Información Nacional de Comercio Exterior SINCE.

Ejecutor: Instituto Colombiano de Comercio Exterior - INCOMEX.

Sistema de Información Biogeográfico en Línea para Colombia.

Ejecutor: Fundación Puerto Rastrojo.

- Se apoya la consolidación de Servicios de Información de valor agregado y sus interfaces con redes nacionales e internacionales.

- Conformación de una Red Nacional de 23 Centros de Gestión "con el fin de establecer un mecanismo común de interconexión y comunicación de la Red Colombiana de Educación, Ciencia y Tecnología, CETCOL". Con esta red se conectan 500 instituciones, ligadas de una $u$ otra manera a la investigación.

Esta red ofrece, entre otros, los siguientes servicios:

- Servicios de información industrial a empresarios sobre materias primas, procesos, noemas técnicas, tecnologías, gestión, etc.

- Atención a consultas: búsquedas de documentos, datos, etc. 
- Diseminación de información a través de boletines periódicos o vía Internet de noticias de interés en estos campos.

- Suministro de fotocopias

- Análisis y síntesis de documentos recientes.

- Recopilación y análisis de datos

- Servicios de consultoría.

CETCOL es administrada por una empresa de derecho privado, la Corporación InteRed. En cuya junta directiva están COLCIENCIAS, ICFES, representantes de universidades y del sector privado.

CETCOL cuenta con un Centro de Gestión, localizado en la $\mathrm{He}$ meroteca Nacional, en Bogotá y 4 centros de Operación regional: Centro de Operación Zona Central, en la Universidad de los Andes, Bogotá; Centro de Operación Zona Sur, ubicado en Cali, Universidad del Valle. Centro de Operación Zona Occidental, asentado en Medellín, Universidad Eafit; Centro de Operación Zona Norte, situado en Barranquilla.

\section{Algunas acciones internacionales}

Como una muestra de las acciones internacionales en el camo de la información, se van a resaltar la participación colombiana en INFOLAC, los esfuerzos de cooperación de la Organización de Estados Iberoamericanos, OEI, para reflexionar sobre los sistemas de información de la subregión andina y el Proyecto de doctorado en Bibliotecología entre la Universidad Carlos III, de Madrid y 2 universidades colombianas.

INFOLAC es el Programa Regional para el Fortalecimiento de la Cooperación entre Redes y Sistemas Nacionales de Información para el Desarrollo en América Latina y el Caribe, impulsado por UNESCO en colaboración, en sus inicios, 1987, con la Comisión Económica Para América Latina y del Caribe, CEPAL. Su influencia ha sido importante en la elaboración de políticas de información en el área de América Latina y el Caribe.

En ese año se definía como «un mecanismo regional de concertación interinstitucional y al mismo tiempo un instrumento catalizador de acciones y proyectos entre redes y sistemas nacionales de información para el desarrollo" (CEPAL/CLADES, UNESCO/PGI, 1987). En la actualidad "tiene como propósitos inmediatos, convertirse en el Grupo Consultivo Subregional en los temas relacionados" y desarrollar el Centro de Vigilia e Inteligencia Tecnológica (COLCIENCIAS, 1996, p. 3). 
Muchas de las políticas regionales en este campo han sido formuladas y recomendadas desde allí.

Por su parte, el año pasado la OEI realizó la Reunión Andina de Centros de Información en Ciencia y Tecnología, en Caracas. Allí se recomend6, entre otras cosas, crear una uespina dorsal de comunicaciones entre los países de la región sobre la base de las estructuras nacionales (IBEROAMERICANA)" y crear en los países donde no existan organismos, como COLCIENCIAS y CONICYT de Venezuela, que coordinen el trabajo para la constitución de redes y sistemas de información por áreas del conocimiento.

Asimismo, existe un Convenio Marco de Cooperación entre la Universidad Carlos III y la Universidad de Antioquia, en Medellín. Aprovechando ese instrumento $\mathrm{y}$ en asocio con la Universidad Javeriana de Bogotá, se proyecta un programa de Doctorado en Bibliotecología, que comenzaría a más tardar en 1998, en las dos universidades colombianas con el fin de impulsar la investigación y capacitar a un buen número de profesionales colombianos en este campo.

\section{Apreciaciones finales}

El país está adquiriendo la estructura telemática suficiente para conectarse internamente y con todo el mundo. Las acciones para introducir telemáticamente los catálogos de todas las bibliotecas universitarias y centros de investigación, y crear un eficiente y adecuado sistema de este tipo que permita la cooperación ágil, marchan pero no a la velocidad requerida. A esto se suma la escasez de recursos y la necesidad de racionalizarlos al máximo. España está en una situación inmejorable al respecto. Posee, de acuerdo con mi experiencia, un sistema de información eficiente y con unos fondos muy importantes en lengua castellana, quizá los más importantes del mundo de habla hispana. El acceso vía Internet permite conocer esos recursos, por lo que sería posible establecer convenios interbibliotecarios de préstamo, fotocopias, etc., con países como el nuestro. Esto multiplicaría geométricamente los recursos bibliográficos en estos estados y de paso contribuiría a crear una cultura científica y una sólida cooperación entre las comunidades científicas en lengua castellana, sin contar con el acceso a los fondos en otras lenguas vía España en la Unión Europea.

Adicionalmente, podría potenciarse la experiencia española para crear sistemas de información dentro de parámetros $\mathrm{I}+\mathrm{D}$ en países y subregiones de América Latina, como la Subregión Andina. Esta coo- 
peración se daría no sólo en el campo de la cooperación académica sino en los de la gestión y el entrenamiento de los soportes técnicos. Hasta ahora, casi todas las políticas en este campo y el entrenamiento provienen de acciones como las de la UNESCO, ya señaladas, y de los Estados Unidos.

\section{Notas}

1 En Colombia es el del porcentaje de docentes universitarios que poseen título de materia y doctorado: sólo el $12,8 \%$ acreditan el primero, y apenas el $2,4 \%$, el segundo. Claro está que hay que tener en cuenta que en las principales universidades del país, aquéllas en que se realizan investigaciones científicas, estos porcentajes varían drásticamente. Como un dato ilustrativo, es importante señalar que en 1996 había en Colombia 26 programas conducentes al título de doctor y en 1998, están previstos 28. Este número aparentemente bajo se debe a que las autoridades académicas han privilegiado la calidad sobre la cantidad, autorizando el funcionamiento de dichos programas luego de una evaluación que incluye árbitros internacionales.

\section{Bibliografía}

InterRed. Plegable Informativo. Santafé de Bogotá, 1996.

Sistemas de información científica y tecnológica. Plegable. COLCIENCIAS 1996.

Carta de Colciencias. Vol 19 No 51996.

Ciencia y tecnología para un desarrollo sostenible y equitativo. Implementación de la Política Nacional de Ciencia y Tecnología: 1994 - 1998. Consejo Nacional de Ciencia y Tecnología, preparado por COLCIENCIAS. Santafé de Bogotá, 20 de junio de 19995. Citado como 1995a.

Plan estratégico de los sistemas de información científica y tecnológica. COLCIENCIAS, Santafé de Bogotá, junio de 1995. Citado como 1995b.

Bases conceptuales e instrumentales del proceso de regionalización de la ciencia y la tecnología. Estrategia de regionalización. COLCIENCIAS. Santafé de Bogotá, junio de 1995.

Proyecto: Red de ciencia, educación y tecnología de Colombia: CETCOL y enlace internacional con INTERNET ICFES - COLCIENCIAS. Icfes, Santafé de Bogotá, D.C., junio de 1994.

El sistema nacional de ciencia y tecnología. Instrumentos Jurídicos. Ciencia y Tecnología para una Sociedad Abierta. Colciencias, Departamento Nacional de Planeación. Santafé de Bogotá, abril de 1991.

CARDonA DE GiL, Bertha Nelly y CAmpuzano, Yolanda: Administración de redes y sistemas de información científica y tecnológica. Universidad del Quindío. Armenia (Col), 1991.

INFOLAC. Informe Final de la Reunión sobre "Los lineamientos y Puesta en Marcha de un Programa Regional para el Fortalecimiento de la Coperación entre Redes y Sistemas Nacionales de Información para el Desarrollo en América Latina y el Caribe" Santiago de Chile, 3 al 7 de noviembre de 1986. CEPAL/CLADES, UNESCO/PGI, Santiago de Chile 1987. 